\title{
Research on Analytic Model of Project Chain Risk Elements Transmission Based on Risk Ranking Method and Data Mining
}

\author{
Cun Bin Li, Wei Dong and Shuai Shuai Lin \\ School of Economic \& Management, North china Electric Power University, \\ Beijing 102206, China \\ E-mail:18811306733@163.com
}

\begin{abstract}
Risk management in project chain or multi-project is becoming a hot spot in the field of project management, however the analysis of project chain risk elements transmission almost stays in the angle of qualitative. So, in order to study the risk transmission process in project chain, the analytic model of project chain risk element transmission was established. In the beginning, the data mining method is introduced to acquire the risk elements transmission matrix and estimate the state of the risk elements through the previous state. Then, for ranking both probabilistic type and fuzs. type risk elements, probability and fuzzy numbers were transformed interval numbers and ranking score was calculated by a ranking method. Finally, through the computation, the key risk element of different states can be obtained, through which decision makers can adjust the distribution of resources and control the kes risk element. The analysis of a case has verified the validity and practicability of this method.
\end{abstract}

Keywords: risk ranking; risk elements transmission, data mining; project chain

\section{Introduction}

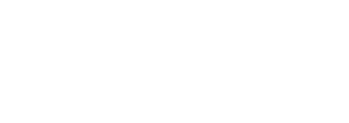

Risk management is one of the most important subjects of project management and is widely used in constraction projects, software projects, finance projects and innovation projects. Although many scholars did a lot of researches on risk management and got many achievements, there is sti much space for the further study of risk management in generalized projects from the aspect of risk element transmission. LI [1-2] considered the transmission effect in generalized projects and proposed the risk elements transmission theory. The theory gave the quantitative study on risk elements and paid more attention to calculating the final risk elements probability distribution from each risk elements probability distribution. However, these literatures were aimed at risk elements transmission of single project. With the rapid development of project management, project chain or multi-project has become a new research tendency. Papers [3-4] presented the concept of multi-project management and gave the qualitative research in practice early. Qi [5] studied on the impact of PMO for multi-project management of contracting construction enterprises based on structural equation model. Zhang [6] researched on game analysis and resources allocation in multi-project management. Jirachai [7] employed liner programming to solve limited resource allocation in multi-mode project under certain periods. George [8] used operation research methods to forecast cash flow in multi-project. Many scholars used intelligent algorithms on resources and periods risk of multi-mode or multi-project, such as differential evolution, heuristic algorithm, tabu search and neural networks. Risk ranking also becomes a hot spot of risk management, Chen [9] presented the fuzzy risk analysis based on new fuzzy ranking methods. Daniel [10] employed the risk ranking to identify the key risk factors in target cost contracts of construction projects. Brito [11] presented the use of risk ranking method in natural gas pipelines project considering decision maker's preferences. Wang [12] analyzed the 
drawbacks of traditional risk priority number and proposed the risk possibility number for more reasonable raking based on maximum entropy theory. All these studies above got many achievements, but some only analyzed risks qualitatively and there were no literatures considering risk elements transmission effect in multi-project, and the critical risks were also not identified effectively. So it is necessary to establish a generalized risk element transmission model in project chain or multi project.

In reference to the papers above, in this paper, we presented a new analytic model of risk element transmission in project chain based on risk ranking method. First, project chain was defined with considering risk element transmission. Two types of risk elements were defined and measured. Then data mining method was used to acquire risk transmission matrix from risk registration database. The probabilistic type and fuzzy type risk elements were transformed to interval numbers for ranking purpose. Through this analytic model, a critical risk path was acquired and the frequency histogram and membership function curve was also presented from the results of an example.

This paper is organized as follows. Section 2 makes a summary of project chain and risk element transmission theory. Section 3 presents the analytic model of risk element transmission in project chain based on risk ranking method and data mining. Anempirical example is presented in Section 4. Section 5 concludes the paper."

\section{Summary of Risk Element Transmission yheory and Rroject Chain}

The transmission effect widely exists in the world such as the butterfly effect. Fluctuation transfers from the source to the end and each middle state is influenced by the transmission process. In order to acquire the transmission process of project risks, the generalized project risk element transmission theory was proposed. The main idea of the theory is the quantitative relationship between risk elements and the final goal of project. The random or non-random variables transferring fluctuation to final goal of project are defined as risk elements. So the transmission process can be described as the fluctuation of risk elements transferring to olher risk elements or project state and finally to the goal of project, for example, construction period of one step delay will lead to delay of other steps even the whole period.

Project chain occurs in enterprise with the increase of investment and productivity. Projects share the investment, labor, and other resources, so resources used in one project occupies the access chance of other projects and lead to increasing opportunity cost. Otherwise, losses will als transfer to other projects because of the relationship of resources sharing. So the projects are connected by all kinds of resources, namely project chain, the resource in project chain can be viewed as the risk elements, as Figure 1 shows.

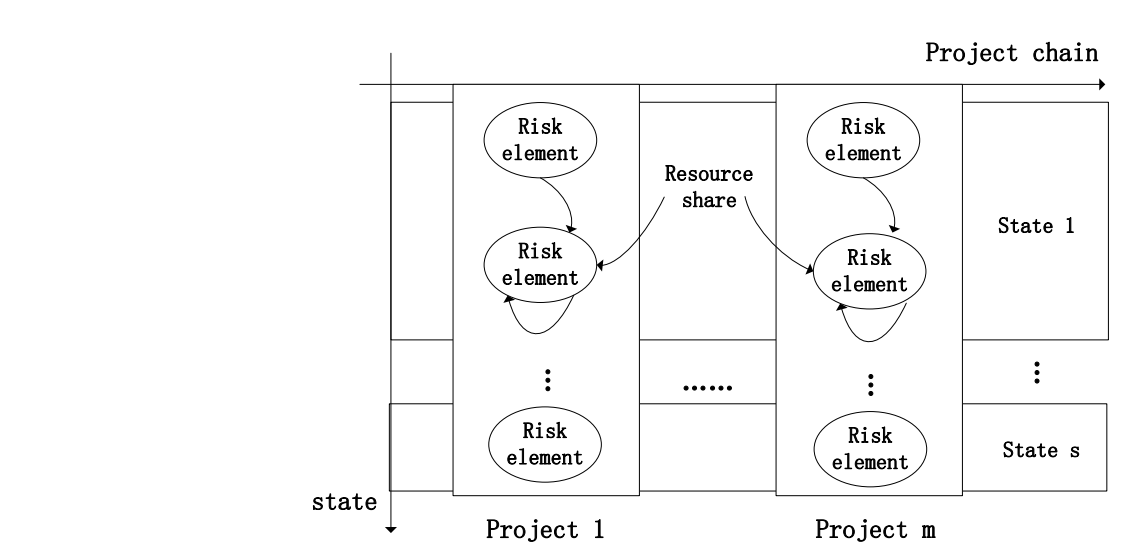

Figure 1. Risk Elements Transmission in Project Chain 
But, to further study, tow problems must be solved:

1) How to represent and measure risk element quantitatively;

2) How to represent the transmission process in project chain.

For the first problem, risk elements can be divided into probabilistic type and fuzzy type from the view of uncertainty. Probabilistic type is described by probability distribution based on mathematical statistics theory and entropy theory and it has the characteristics of objectivity. Probabilistic type risk elements are always used to describe the random event occurring in the transmission process. Incorporated with entropy theory, Probabilistic type risk element is defined as a random event and can be measured as follow

$$
F(x)=-\int_{-\infty}^{+\infty} f(x) \log f(x) d x
$$

Where ${ }^{x}$ denotes the continuous probabilistic type risk element and $f(x)$ is the probability density function; $F(x)$ denotes the average information gained from the random event. For the discrete probabilistic type, risk element is measured as follow

$$
F\left(p_{e}\right)=-K \sum_{i=1}^{n} p_{e}^{i} \log p_{e}^{i}
$$

Where $\mathrm{K}$ is a positive constant and $p_{e}^{i}$ denotes the probability of risk element event; $\sum_{i=1}^{n} p_{e}^{i}=1$

. The base of logarithms are 2,e and 10; the unit of information are Bit, Nat and Dit respectively. Under most situation, it is hard to estmate the appropriate distribution and the objective data, so the fuzz lnguistic terms are always used in estimate the subjective event. Let $\tilde{A}$ be a sub fưzzy set of risk element universe of $\operatorname{discourse} S$. Any fuzzy type risk element ${ }^{x_{i}}$ belongs to $S$. So the fuzzy risk element can be represent by

$$
\tilde{A}=\sum_{i=1}^{n} \mu_{i} / x_{i}
$$

Where $\mu_{i} \in[\beta, 1]$ denotes the membership function of the fuzzy risk element. Fuzzy type risk element can be also represented by a generalized trapezoidal fuzzy number $\tilde{A}=(a, b, c, d$; $(v)$ as shown in Figure.2. If $w=1$, it becomes a normal trapezoidal fuzzy number.
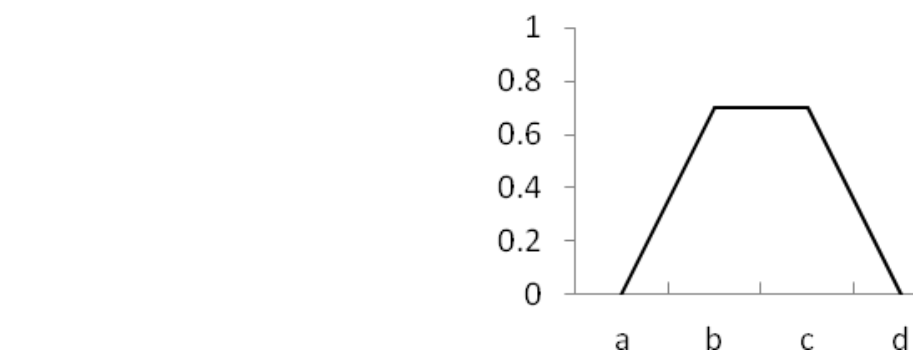

Figure 2. Membership Function of Generalized Fuzzy Number

So risk elements are described and measured by the probabilistic type using probability distribution and the fuzzy type using fuzzy number. For solving the second problem how to represent the transmission process in project chain, an analytic model is constructed in 


\section{Section 3.}

\section{Analytic Model of Risk Elements Transmission in Project Chain}

Transmission processes in project chain are accompanied with resource constrains. Resources used in each project connect the project chain and form the risk state of whole project chain. With the consumption of resources, risk state changes under the risk element transmission effect. In this section, the transmission process leading to changes of risk state will be presented. Data mining and risk ranking methods are employed to establish the analytic model of risk elements transmission in project chain.

\subsection{Definitions of Analytic Model}

In project chain, risk states of each project are affected by the resource allocation and other risk factors which can be defined as risk elements. It is necessary to find out how these risk elements affect each other and lead to the risk state of project. The definitions of the model are as follows.

Definition 1 State set of project chain $S=\{s \mid 0 \leq s \leq n\}$, denotes the different state or stage of project chain divided by the consumption of resources.

Definition 2 Risk elements set $R=\left\{r_{i}, \tilde{r}_{j} \mid 0 \leq i \leq m, 0 \leq j \leq n-m\right\}$, denotes all risk elements caused by the resource allocation or other risk factors in project chain; ${ }^{{ }^{i}}$ means a probabilistic risk element and $\tilde{r}_{j}$ means a fuzzy risk elemen?

Definition 3 Risk elements transmission matrix $A^{s}=\left(a_{i j}^{s}\right)_{n \times n}$, where $a_{i j}^{s}$ denotes the transmission probability from $k_{i}$ to between state $S_{\text {and }} s+1$.

Definition 4 Possibility degtee(PD) of interval number. Assume $a=\left[a^{-}, a^{+}\right]$and $b=\left[b^{-}, b^{+}\right]$are two interal numbers, and $\omega(a)=a^{+}-a^{-}$, so the possibility degree of $a \geq b$ is presented as follow

Where

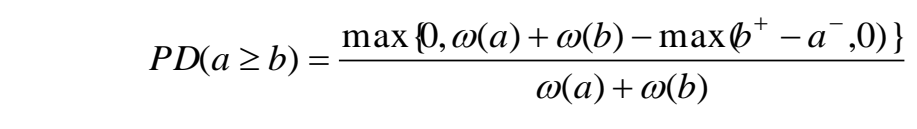

Otherwise, two fuzzy arithmetic operations are defined as follows to calculate the fuzzy type isk elements. Assume risk elements $\tilde{r}_{i}=\left(a_{i}, b_{i}, c_{i}, d_{i} ; w_{i}\right)$ and $\tilde{r}_{j}=\left(a_{j}, b_{j}, c_{j}, d_{j} ; w_{j}\right)$ are tow generalized trapezoidal fuzzy numbers, and $\lambda$ means any real numbers between 0 and 1 .

Definition 5 Fuzzy arithmetic operation ${ }^{\otimes}$ :

$$
\begin{aligned}
\lambda \otimes \widetilde{r}_{j} & =\lambda \otimes\left(a_{i}, b_{i}, c_{i}, d_{i} ; w_{i}\right) \\
& =\left(\lambda \times a_{i}, \lambda \times b_{i}, \lambda \times c_{i}, \lambda \times d_{i} ; w_{i}\right)
\end{aligned}
$$

Definition 6 Fuzzy arithmetic operation $\oplus$ :

$$
\begin{aligned}
\tilde{r}_{i} \oplus \tilde{r}_{j} & =\left(a_{i}, b_{i}, c_{i}, d_{i} ; w_{i}\right) \oplus\left(a_{j}, b_{j}, c_{j}, d_{j} ; w_{j}\right) \\
& =\left(a_{i}+a_{j}, b_{i}+b_{j}, c_{i}+c_{j}, d_{i}+d_{j} ; \max \left(w_{i}, w_{j}\right)\right)
\end{aligned}
$$




\subsection{Data Mining for Preparation}

Data mining is widely used in project management to acquire the transcendental and objective data from historical database. In the analytic model, the risk elements and transmission matrix are got from historical database by follow steps.

Step 1: Establish a risk register database. Record the historical data observed from similar project chain. Each record contains the risk elements occurred and the consumption state of resources.

Step 2: Pretreat the database and select the distinct state into state set $S$.

Step 3: Select all risk elements occurred in one or more state into risk elements set $R$, and acquire the transmission matrix from the database by using the follow method:

for $s=1$ to $\mathrm{m}$ and $i, j=1$ to $\mathrm{n}$

Select count( $\left.{ }^{r_{j}}\right)$ as $\operatorname{num}\left(r_{j} \mid r_{i}, s\right)$ from database where risk element ${ }^{r_{i}}$ occurred ând the state is $s$.

Select count $\left(r_{i}\right)$ as $\operatorname{num}\left(r_{i} \mid s\right)$ from database where risk element ${ }^{r_{i}}$ occurred and the state is $s$.

Then calculate the transmission probability $a_{i j}^{s}$ as follow:

$$
a_{i j}^{s}=\frac{\operatorname{num}\left(r_{j} \mid r_{i}, s\right)}{\operatorname{num}\left(r_{i} \mid s\right)}
$$

\subsection{Risk Ranking Method for Analytic Model}

Based on the data mining method ane state set, $\mathbb{S}=\{\mid 0 \leq s \leq l\}$ and the transmission matrix $A^{s}$ can be acquired. For quantitative representing, divide the risk elements set $R=\left\{R^{\prime}, R^{\prime \prime}\right\}$ into probabilistlc type and fuzzy type. The calculation steps of the analytic model are shown as follows:

Step 1: For probabilistic type rîsk elements $R^{\prime}=\left\{r_{i}, 1 \leq i \leq m\right\}$, acquire the initial probability $P_{1}\left(r_{i}\right)$ from risk regster database when state $s=1$.

$$
\bigcirc \quad P_{1}\left(\odot=\frac{\operatorname{num}\left(r_{i} \mid s=1\right)}{\operatorname{num}(R \mid s=1)}\right.
$$

Where $\operatorname{num}(R \mid s \in \mathbb{O}$ denotes the total number of risk elements occurred in the first state.

For fuzzy type risk elements $R^{\prime \prime}=\left\{\tilde{r}_{j}, m+1 \leq j \leq n\right\}$, set the initial fuzzy number by using the fuzzy linguistic term, as Table 1 shows.

\section{Table 1. Linguistic Terms and Relevant Fuzzy Numbers}

\begin{tabular}{cc}
\hline Linguistic terms & Fuzzy numbers \\
\hline Very-low & $(0.09,0.17,0.23,0.28 ; 1.0)$ \\
low & $(0.2,0.26,0.3,0.44 ; 1.0)$ \\
medium & $(0.4,0.48,0.57,0.65 ; 1.0)$ \\
high & $(0.63,0.7,0.75,0.89 ; 1.0)$ \\
Very-high & $(0.86,0.9,0.94,1 ; 1.0)$ \\
\hline
\end{tabular}

Step 2: For each state $s>1$, calculate the new probability $P_{s}\left(r_{i}\right)$ of probabilistic risk elements. 


$$
P_{s}\left(r_{i}\right)=\sum_{j=1}^{m} a_{j i}^{s-1} P_{s-1}\left(r_{j}\right)
$$

Where $a_{j i}^{s-1}$ denotes the transmission probability from ${ }^{r_{j}}$ to ${ }^{r_{i}}$ between state $s-1$ and $s$, and $1 \leq i, j \leq m$.

Aggregate the new fuzzy risk elements $\tilde{r}_{j}$ based on the fuzzy risk elements in state $s-1$ and fuzzy number arithmetic operation defined in Eqs.(5)-(6).

$$
\tilde{r}_{j}^{s}=\left(a_{1 j} \otimes \tilde{r}_{1}^{s-1}\right) \oplus\left(a_{2 j} \otimes \tilde{r}_{2}^{s-1}\right) \oplus \cdots \oplus\left(a_{m j} \tilde{r}_{m}^{s-1}\right), m+1 \leq j \leq n
$$

Then transform $\tilde{r}_{j}^{s}$ into a standardized fuzzy number $\tilde{r}_{j}^{s^{*}}$ based on Chen\&chenc'method.

$$
\tilde{r}_{j}^{s^{*}}=\left(\frac{a_{j 1}}{K}, \frac{a_{j 2}}{K}, \frac{a_{j 3}}{K}, \frac{a_{j 4}}{K} ; w_{j}\right)
$$

Where $\quad \underset{i, j}{\left.K=\max \nmid\left|a_{i j}\right| \mid 1\right)},\left|a_{i j}\right|_{\text {denotes the absolute value and }}$ the upper bound of $\left|a_{i j}\right|, 1 \leq i \leq n-m$ and $1 \leq j \leq 4$.

Step 3: Transform the probabilistic risk elements and fuzzy risk elements into the interval numbers in each state $\mathrm{s}$.

For probabilistic risk element $r_{i}^{s}$, calcuate the average probability $\bar{P}\left(r_{i}\right)$ and standard deviation $S T D\left(r_{i}\right)$, then calculate the gelevant interval numbers $u_{i}^{s}=\left[u_{i}^{s-}, u_{i}^{s+}\right]$ as follows:

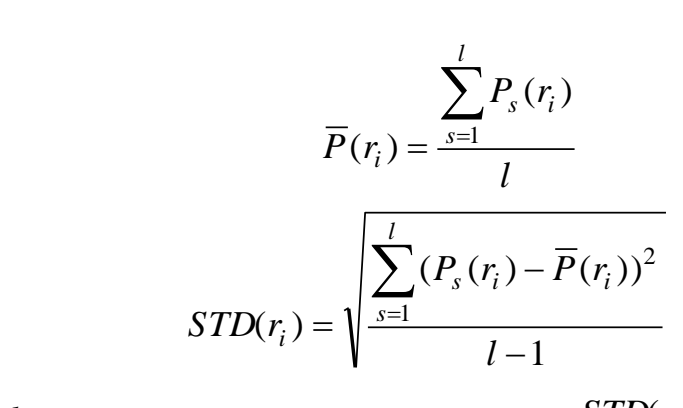

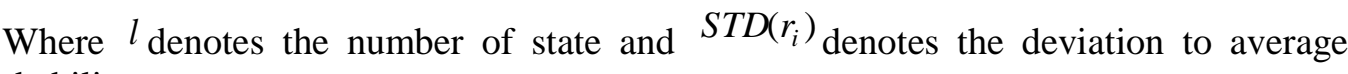
probability.

$$
\left\{\begin{array}{l}
u_{i}^{s-}=r_{i}^{s}-S T D\left(r_{i}\right) \\
u_{i}^{s+}=r_{i}^{s}+\operatorname{STD}\left(r_{i}\right)
\end{array}, 1 \leq i \leq m\right.
$$

Respectively, for the standardized fuzzy risk element $\tilde{r}_{j}^{s^{*}}=\left(a_{j 1}, a_{j 2}, a_{j 3}, a_{j 4} ; w_{j}\right)$, calcolate the interval numbers by $\alpha$-cut .The value of $\alpha$ denotes the decision maker's attitude to the state; the larger the value of $\alpha$, the more attention was paid to this state. So the interval number $u_{j}^{s}=\left[u_{j}^{s-}, u_{j}^{s+}\right]$, is calculate as follow.

$$
\left\{\begin{array}{l}
u_{j}^{s-}=a_{j 1}+\frac{\left(a_{j 2}-a_{j 1}\right) \times \alpha}{w_{j}} \\
u_{j}^{s+}=a_{j 4}-\frac{\left(a_{j 4}-a_{j 3}\right) \times \alpha}{w_{j}}
\end{array}, m+1 \leq j \leq n\right.
$$

And the $\alpha_{\text {cut set }} \tilde{r}_{j \alpha}^{s^{*}}$ of fuzzy risk element $\tilde{r}_{j}^{s^{*}}$ is shown respectively as follow. 


$$
\tilde{r}_{j}^{s^{*}}=\left\{\begin{array}{l}
\alpha, r_{j} \in\left[u_{j}^{s-}, u_{j}^{s+}\right] \\
0, r_{j} \notin\left[u_{j}^{s-}, u_{j}^{s+}\right]
\end{array}\right.
$$

Step 4: Rank the interval numbers $\left\{u_{i} \mid 1 \leq i \leq n\right\}$ in each state $s$, where $1 \leq s \leq l$. There are $n$ interval numbers which consist of $m$ probabilistic risk elements interval numbers and $n-m_{\text {fuzzy risk elements interval numbers. In a certain state }}{ }$, Calculate the possibility degree matrix (PDM) of all $n$ interval numbers,

$$
P D M^{s}=\left(p d_{i j}^{s}\right)_{n \times n}
$$

Where $p d_{i j}=P D\left(u_{i}^{s} \geq u_{j}^{s}\right)_{\text {based on Eq.(4). Then calculate the rank score of each }}$ interval numbers as follow.

$$
\operatorname{Score}\left(r_{i}^{s}\right)=\frac{\sum_{j=1}^{n} p d_{i j}^{s}+\frac{n}{2}-1}{n \times(n-1)}, i=1 . . n, n \geq 2
$$

Where $n$ denotes the number of all risk elements. The Targer the value of $\operatorname{Score}\left(r_{i}^{s}\right)$, the better the rank of risk element $r_{i}$ in state ${ }^{S}$, and the risk element $r_{i}$ is more likely to $\sum_{\text {occur. }}^{n} p d_{i j}^{s}$ denotes the sum possibility degree of the inferyal number $u_{i}^{s}$ larger than other interval numbers in state $s$. The maximum and mininum score is decided by the number $n$.

Based on the steps above, choose the hisk elements that have maximum rank score as the critical nodes. Then connect these nodes to be a critical risk path. Otherwise, based on Eq.(9), the probability distribution of probabilistic type risk elements can be shown as the frequency histogram. Respectivery, the membership function of fuzzy type risk elements can be acquired.

\section{Example Analysis}

In this section, we apply the analytic model to deal with risk analysis problem in manufacture enterprise. A nanufacturer always has multiple production lines, which share resources of enterprise, such as labor, cash flow, raw materials. Decision maker wants to know whether the resøyrces allocation plan is appropriate so that he can adjust resources allocation to fulfill the production goals. Let $L_{1}, L_{2}, L_{3}$ represent three production lines and $r_{1}, \ldots$ denote six risk elements caused by six resources allocation. Decision maker divides the whole production cycle into five states by referencing the key points of esources allocation. During the six risk elements, $r_{1}, r_{2}, r_{3}$ are the probabilistic type risk elements and $r_{4}, r_{5}, r_{6}$ are the fuzzy type risk elements. Decision maker defines the linguistic term set to give the initial fuzzy number of fuzzy risk elements.

Based on data mining method and linguistic terms set, the initial values of six risk elements set are shown as Table 2 and the risk elements transmission matrix $A^{1}, A^{2}, A^{3}, A^{4}$ are shown in Table 2 .

\section{Table 2. Initial Value of Risk Elements}

\begin{tabular}{cc}
\hline Risk elements & Initial value \\
\hline $\mathrm{r}_{1}$ & 0.3 \\
\hline
\end{tabular}




\begin{tabular}{cc}
\hline $\mathrm{r}_{2}$ & 0.5 \\
$\mathrm{r}_{3}$ & 0.7 \\
$\mathrm{r}_{4}$ & $(0.2,0.26,0.3,0.44 ; 0.8)$ \\
$\mathrm{r}_{5}$ & $(0.86,0.9,0.94,1 ; 0.6)$ \\
$\mathrm{r}_{6}$ & $(0.4,0.48,0.57,0.65 ; 0.7)$ \\
\hline
\end{tabular}

According to the data mining method and Eq.(7), the risk element transfer matrix of different states can be acquired, as Table 3 shows.

Table 3. Risk Elements Transmission Matrix $A^{1}, A^{2}, A^{3}, A^{4}$

\begin{tabular}{ccccccc|ccccccc}
\hline $\mathrm{A}^{1}$ & $\mathrm{r}_{1}$ & $\mathrm{r}_{2}$ & $\mathrm{r}_{3}$ & $\mathrm{r}_{4}$ & $\mathrm{r}_{5}$ & $\mathrm{r}_{6}$ & $\mathrm{~A}^{2}$ & $\mathrm{r}_{1}$ & $\mathrm{r}_{2}$ & $\mathrm{r}_{3}$ & $\mathrm{r}_{4}$ & $\mathrm{r}_{5}$ & $\mathrm{r}_{6}$ \\
\hline $\mathrm{r}_{1}$ & 0.4 & 0.44 & 0.16 & 0 & 0 & 0 & $\mathrm{r}_{1}$ & 0.36 & 0.18 & 0.46 & 0 & 0 & 0 \\
$\mathrm{r}_{2}$ & 0.21 & 0.53 & 0.26 & 0 & 0 & 0 & $\mathrm{r}_{2}$ & 0.48 & 0.25 & 0.27 & 0 & 0 & 0 \\
$\mathrm{r}_{3}$ & 0.43 & 0.21 & 0.36 & 0 & 0 & 0 & $\mathrm{r}_{3}$ & 0.39 & 0.28 & 0.33 & 0 & 0 & 0 \\
$\mathrm{r}_{4}$ & 0 & 0 & 0 & 0.55 & 0.1 & 0.35 & $\mathrm{r}_{4}$ & 0 & 0 & 0 & 0.55 & 0.3 & 0.15 \\
$\mathrm{r}_{5}$ & 0 & 0 & 0 & 0.2 & 0.6 & 0.2 & $\mathrm{r}_{5}$ & 0 & 0 & 0 & 0.1 & 0.7 & 0.2 \\
$\mathrm{r}_{6}$ & 0 & & 0 & 0.16 & 0 & 0.84 & $\mathrm{r}_{6}$ & 0 & 0 & 0 & 0.19 & 0.17 & 0.64 \\
\hline $\mathrm{A}^{3}$ & $\mathrm{r}_{1}$ & $\mathrm{r}_{2}$ & $\mathrm{r}_{3}$ & $\mathrm{r}_{4}$ & $\mathrm{r}_{5}$ & $\mathrm{r}_{6}$ & $\mathrm{~A}^{4}$ & $\mathrm{r}_{1}$ & $\mathrm{r}_{2}$ & $\mathrm{r}_{3}$ & $\mathrm{r}_{4}$ & $\mathrm{r}_{5}$ & $\mathrm{r}_{6}$ \\
\hline $\mathrm{r}_{1}$ & 1 & 0 & 0 & 0 & 0 & 0 & $\mathrm{r}_{1}$ & 0.66 & 0.1 & 0.24 & 0 & 0 & 0 \\
$\mathrm{r}_{2}$ & 0.2 & 0.62 & 0.18 & 0 & 0 & 0 & $\mathrm{r}_{2}$ & 0.21 & 0.32 & 0.47 & 0 & 0 & 0 \\
$\mathrm{r}_{3}$ & 0.29 & 0.16 & 0.55 & 0 & 0 & 0 & $\mathrm{r}_{3}$ & 0.21 & 0.21 & 0.58 & 0 & 0 & 0 \\
$\mathrm{r}_{4}$ & 0 & 0 & 0 & 0.32 & 0.17 & 0.51 & $\mathrm{r}_{4}$ & 0 & 0 & 0 & 0.55 & 0.1 & 0.35 \\
$\mathrm{r}_{5}$ & 0 & 0 & 0 & 0.33 & 0.67 & 0 & $\mathrm{r}_{5}$ & 0 & 0 & 0 & 0.1 & 0.8 & 0.1 \\
$\mathrm{r}_{6}$ & 0 & 0 & 0 & 0.62 & 0 & 0.38 & $\mathrm{r}_{6}$ & 0 & 0 & 0 & 0.39 & 0.19 & 0.58 \\
\hline
\end{tabular}

Based on Eq.(9)-(11), calculate the new probabilit and the new fuzzy number of risk elements from state 2 to state 5. Decision maker's attitudes to each state $\operatorname{are}^{\alpha}=\{0.4,0.7,0.5,0.6,0.8\}$; base on Eq.(12)-(15), calculate the corresponding interval numbers of each risk elements from state 2 to state 5 . Then calculate the possibility degree of interval numbers based on Eq.(4) and rank the interval numbers based on Eqs(17)-(18). The results of each state are shown in Table 4.

Table 4. Results of State 2 to State 5 of the Manufacture Enterprise Example






\begin{tabular}{|c|c|c|c|c|c|c|}
\hline 4 & $(0.632,0.713,0.79,0.912 ; 1)$ & {$[0.68,0.839]$} & $\begin{array}{c}0.229 \\
4\end{array}$ & $(0.541,0.611,0.678,0.786 ; 1$ & {$[0.597,0.699$} & $\begin{array}{c}0.215 \\
1\end{array}$ \\
\hline 5 & $(0.447,0.491,0.532,0.605 ; 1$ & {$[0.474,0.562$} & $\begin{array}{c}0.174 \\
1\end{array}$ & $(0.493,0.547,0.598,0.684 ; 1$ & {$[0.536,0.632$} & $\begin{array}{c}0.164 \\
3\end{array}$ \\
\hline 6 & $\begin{array}{c}(0.381,0.436,0.488,0.572 ; 1 \\
)\end{array}$ & $\begin{array}{l}{[0.414,0.522} \\
]\end{array}$ & $\begin{array}{c}0.151 \\
8\end{array}$ & $\begin{array}{c}(0.487,0.551,0.613,0.712 ; 1 \\
)\end{array}$ & $\begin{array}{l}{[0.539,0.632} \\
]\end{array}$ & $\begin{array}{c}0.172 \\
5\end{array}$ \\
\hline
\end{tabular}

From the results, the ranking of risk elements in each state are shown; ranking in state 2 is $r_{6}>r_{5}>r_{2}>r_{1}>r_{3}>r_{4}$; ranking in state 3 is $r_{6}>r_{5}>r_{1}>r_{3}>r_{4}>r_{2}$; ranking in state 4 is $r_{1}>r_{4}>r_{5}>r_{6}>r_{3}>r_{2}$ and ranking in state 5 is $r_{4}>r_{1}>r_{6}>r_{5}>r_{3}>r_{4}$. So the critical risk path is $r_{6}-r_{6}-r_{1}-r_{4}$, indicating the critical risk should be paid more attention to avoid risks of resources allocation. It shows that the fuzzy risk element of $r_{4}$ changes sharply from state 3 to state 4 because of the increased impact transferred from $r_{6}$ in state 3 . And from the whole life cycle of production, the probability dist ibution histogram of probabilistic type risk elements is shown in Figure 3.

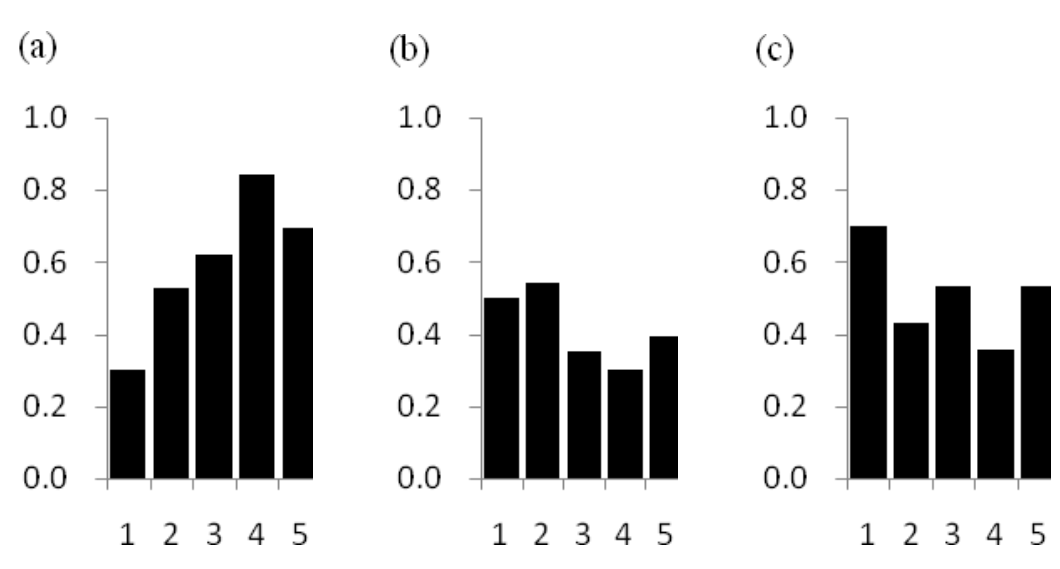

Figure 3 Nistogram of Probabilistic Risk Elements:(a) $r_{1},(b) r_{2},(c) r_{3}$

Respectively, the membersip function curves are shown in Figure 4.

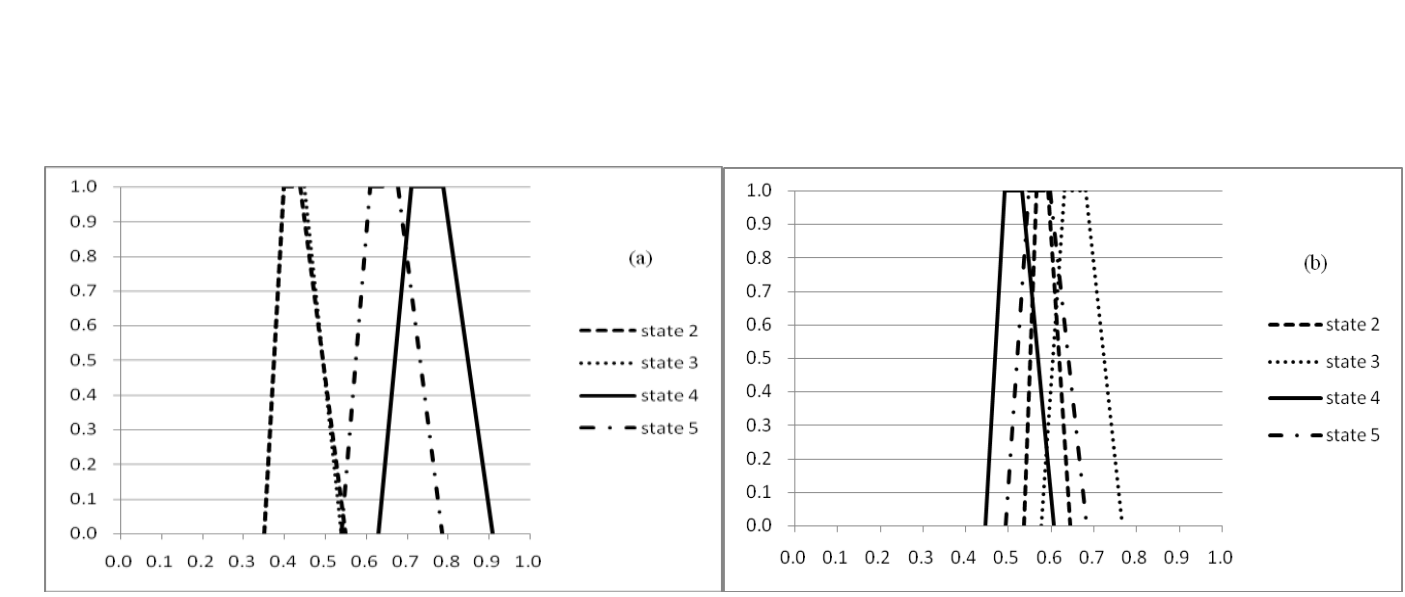




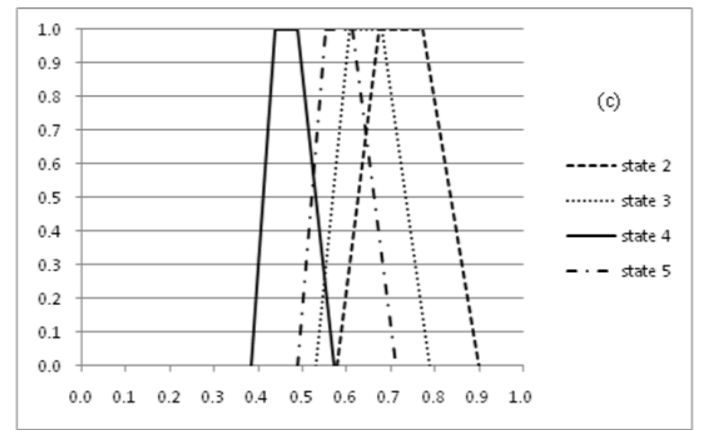

\section{Figure 4. Membership Function Curve of Fuzzy Type Risk Elements:(a) $\mathbf{r}_{4},(b) r_{5},(c) r_{6}$}

\section{Conclusions}

1) Through the model, the key risk route can be calculated, and in order to complete the overall goal of the project on schedule, decision makers can adjust the distribution of resources and control the key risk element according to the critical risk path

2) The generalized risk elements definitions of probabilistic type and fuzzy type are given, and generalized fuzzy numbers are used to measure the fuzzy type risk elements. The data mining method is given to acquire the risk elements transmission matrix.

3) The analytic model of risk elements transmission in froject is presented. Based on the risk elements transmission matrix, two types of risk elements are estimated. In order to rank both probabilistic type and fuzzy type risk tements, the probability and fuzzy numbers are transformed to interval numbers; the rank method is presented to find the critical risk path. Finally, the model is applied to deal with a manufacture enterprise example. During the risk transmission proces $S$ risk elements changes and the critical risk nodes are presented. The frequency histogram of probabilistic type risk elements and membership function cur e of fuzzy type risk elements are also given.

\section{Acknowledgements}

This research is partially funded by the National Natural Science Foundation of China (71271084), and Science and Technology Project of SGCC (521820140017).

\section{References}

[1] L. C. Bin and W. K. Cheng, "The risk element transmission theory research of multi-objective risk-time-cost trade-off”, Journal Computers and Mathematics with Applications, vol. 57, (2008), pp. $1792-1799$.

[2] L. C. Bin and W. J. Jun, “A Model of Generic Project Risk Element Transmission Theory Based on Data Mining”, Journal of Central South University of Technology, vol. 15, no. 1, (2008), pp. 132-135.

[3] A. Jerbrant, "A maturation model for project-based organizations-with uncertainty management as an ever-present multi-project management focus", South African Journal of Economic and Management Sciences, vol. 17, no. 1, (2014), pp. 33-51.

[4] M. C. J. Canië ls and R. J. J. M. Bakens, "The effects of Project Management Information Systems on decision making in a multi project environment”, International Journal of Project Management, vol. 30, no. 2, (2012), pp. 162-175.

[5] S. J. Qi, Y. B. Zhang and J. J. Wu, "Study on the Impact of PMO for Multi-Project Management of Contracting Construction Enterprises-Based on Structural Equation Model", Journal Applied Mechanics and Materials, vol. 584, (2014), pp. 2239-2245.

[6] Q. Zhang and H. B. Chen, "Research on Game Analysis and Resources Allocation in Multi-Project Management”, Journal Applied Mechanics and Materials, vol. 121, (2012), pp. 4619-4624.

[7] J. Buddhakulsomsiri and D. S. Kim, "Properties of multi-mode resource-constrained project scheduling problems with resource vacations and activity splitting", European Journal of Operational Research, vol. 175, no. 1, (2006), pp. 279-295. 
[8] G. Mavrotas, Y. Caloghirou and J. Koune, "A model on cash flow forecasting and early warning for multi-project programmes: application to the Operational Programme for the Information Society in Greece”, International Journal of Project Management, vol. 32, no. 2, (2005), pp. 121-133.

[9] S. M. Chen and K. Sanguansat, "Analyzing fuzzy risk based on a new fuzzy ranking method between generalized fuzzy numbers", Journal Expert Systems with Applications, vol. 38, no. 3, (2011), pp. 2163-2171.

[10] D. W. M. Chan, A. P. C. Chan, P. T. I. Lam, J. F. Y. Yeung and J. H. L. Chan, "Risk ranking and analysis in target cost contracts: Empirical evidence from the construction industry", International Journal of Project Management, (2010).

[11] A. J. Brito and A. T. de Almeida, "Multi-attribute risk assessment for risk ranking of natural gas pipelines”, Journal Reliability Engineering \& System Safety, vol. 94, no. 2, (2009), pp. 187-198.

[12] W. G. Bao, H. H. Zhong and Z. X. Ling, "Risk Possibility Number-A New Model for Risk Evaluation and Prioritization Based on Maximum Entropy Theory", Journal Acta Aeronautica Et Astronautica Sinica, vol. 30, no. 9, (2009), pp. 1683-1689.

\section{Authors}

CunBin Li, professor, doctoral supervisor, Engaged in information risk management and decision, project risk management, and other aspects of this field, School of Economics and Management, North China Electric Power University, Beijing 102206, China, E-mail: Lcb999@263.net.

Wei Dong, Doctoral student, Engaged in Electric power operation and intelligent engineering, and other aspects of this field, School of Economics and Management, North China Electric Power University, Beijing 102206, China, E-mail: dongwei3@sgepri.sgcc.com.cn.

ShuaiShuai Lin, Corresponding author Postgraduate student, Engaged in information risk management and decision and ôther aspects of this field, School of Economics and Management, North China Electrie Power University, Beijing 102206, China, E-mail: 18811306733@163.com.

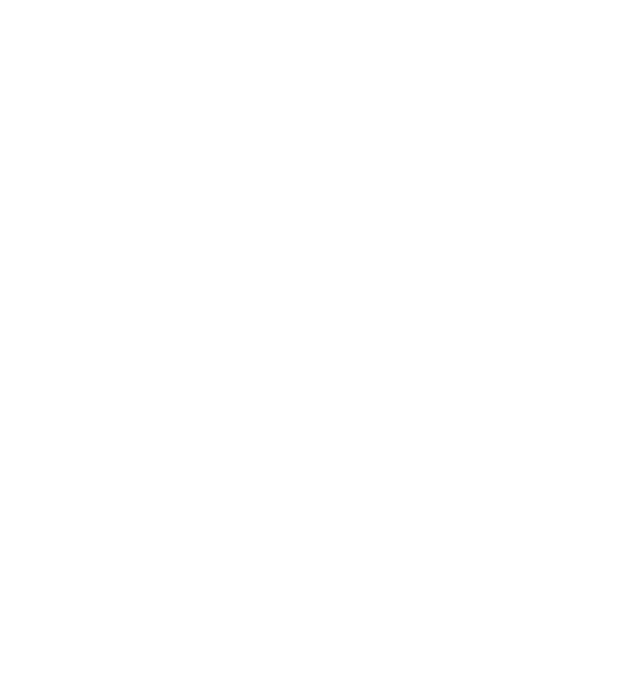


International Journal of Multimedia and Ubiquitous Engineering

Vol.11, No.11 (2016)

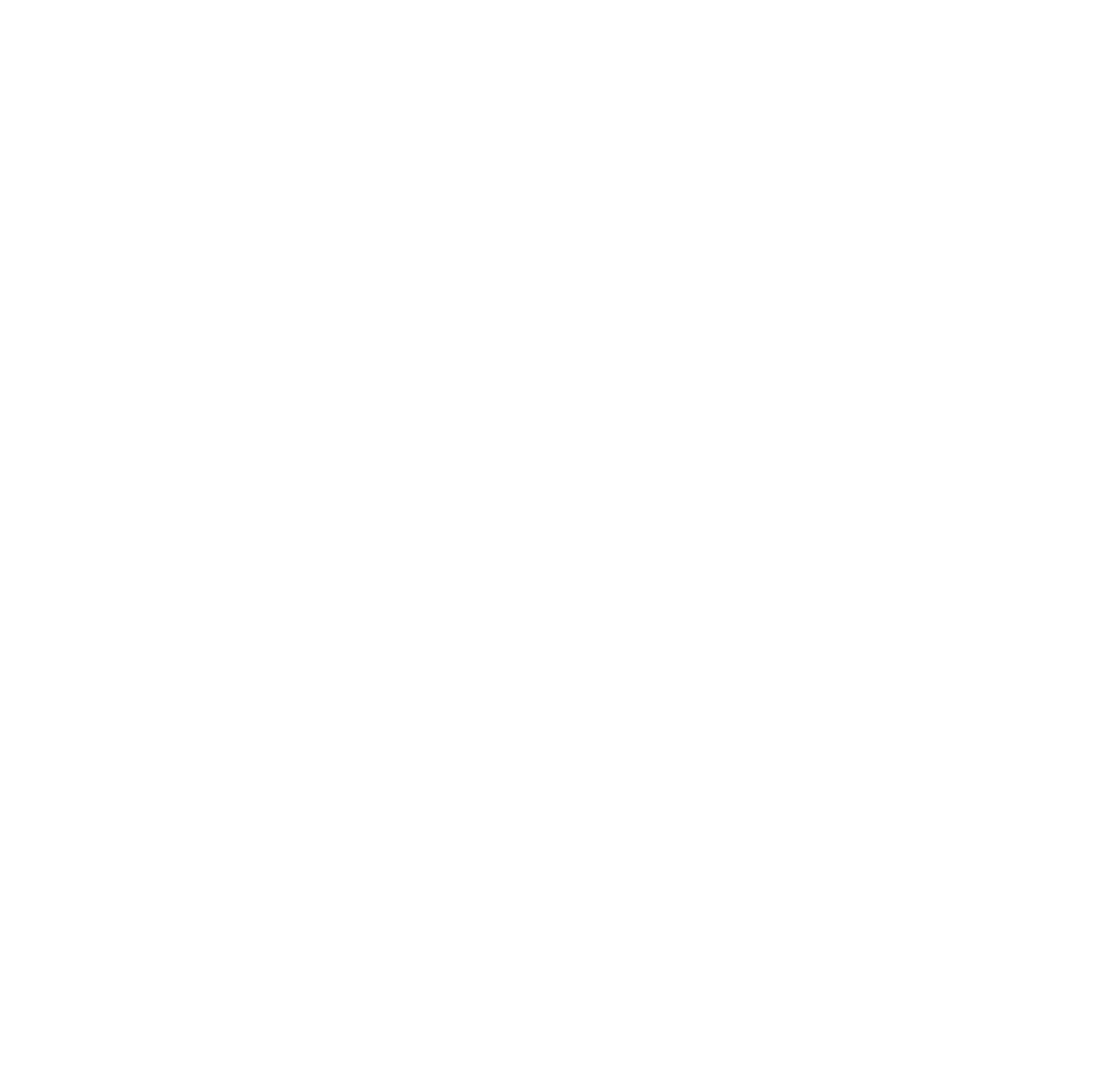

